\section{Mudanças no consumo alimentar de nipo-brasileiros residentes em Bauru, São Paulo, Brasil, 1993-2000}

\author{
Changes in dietary intake among \\ Japanese-Brazilians in Bauru, \\ São Paulo, Brazil, 1993-2000
}

\author{
1 Faculdade de Saúde \\ Pública, Universidade de \\ São Paulo, São Paulo, Brasil. \\ 2 Escola Paulista de \\ Medicina, Universidade \\ Federal de São Paulo, \\ São Paulo, Brasil. \\ 3 Outros membros listados \\ ao final do artigo. \\ Correspondência \\ M. A. Cardoso \\ Departamento de Nutrição, \\ Faculdade de Saúde Pública, \\ Universidade de São Paulo. \\ Av. Dr. Arnaldo 715, \\ São Paulo, SP \\ 01246-904, Brasil. \\ marlyac@usp.br
}

\begin{abstract}
Cross-sectional surveys were performed in 1993 and 2000 on Japanese-Brazilians $(n=328)$ of both sexes, aged 40 to 79 years in 1993, living in Bauru, São Paulo State. Both surveys examined food intake using food frequency questionnaires. Dietary intake in both surveys was compared to Wilcoxon tests according to gender ( $p<$ $0.05)$. Calories from dietary fat increased by $16 \%$ in both genders (mean values in 2000: $31.5 \%$ for women and $30.8 \%$ for men). When the surveys were compared, the main dietary changes were: (a) a 40\% increase in oleic and linoleic acids and calories from fat and (b) a 20\% reduction in dietary cholesterol in both sexes. Among women, an increase in total calorie intake with reduced consumption of vegetables and calories from sweets were observed. Despite increased intake of total dietary fat, there was a decrease in cholesterol intake and an increase in oleic and linoleic acids, fruit, and calcium. However, calcium and vegetable consumption is still short of international recommendations.
\end{abstract}

Food Comsumption; Nutrition; Nutrition Surveys

\author{
Teresa Gontijo de Castro ${ }^{1}$ \\ Carla Novaes Bertolino 1 \\ Suely Godoy Agostinho Gimeno 2 \\ Marly Augusto Cardoso 1 \\ Grupo de Estudo de Diabetes \\ de Nipo-Brasileiros de Bauru 3
}

\section{Introdução}

A avaliação do consumo alimentar habitual tem importante papel na identificação de fatores de risco para doenças crônicas não transmissíveis, sendo fundamental em estudos de comparações entre populações que migraram para países com padrões alimentares, de morbidade e mortalidade distintos de seu país de origem 1 .

No Brasil, estudo de seguimento da comunidade nipo-brasileira de Bauru, Estado de São Paulo, observou aumento na prevalência geral de diabetes do tipo 2 entre os imigrantes e seus descendentes, de $22,6 \%$ em 1993 para $36,2 \%$ em 2000 2. Esta prevalência foi maior que a observada entre os japoneses residentes no Japão, estimada em $7 \% 3$, e também na população brasileira em geral, estimada em $7,6 \%$ entre homens e mulheres de 30 a 69 anos 4.

As diferenças nos perfis de morbidade e mortalidade observadas entre os japoneses e os nipo-brasileiros podem ser explicadas, ao menos em parte, pelas mudanças no padrão alimentar decorrentes da migração. Alimentos tradicionalmente consumidos no Japão, como soja e derivados, peixe e chá verde, têm sido apontados como fatores de proteção para doença coronária isquêmica, enquanto o consumo de frutas frescas (muito consumida pelos nipobrasileiros em relação aos japoneses) pode prevenir a ocorrência de acidentes vasculares e câncer de estômago 5 . Alto consumo de gordu- 
ra total e carboidratos refinados da dieta foi recentemente associado ao risco de síndrome metabólica 6 e distúrbios da homeostase glicêmica 7 , respectivamente, em nipo-brasileiros.

Inquéritos sobre consumo alimentar habitual individual constituem a forma ideal para se caracterizar hábitos alimentares e sua evolução ao longo do tempo em uma população 8 . A descrição das mudanças do consumo alimentar em população de imigrantes permite investigar tendências de comportamento que possam estar associadas ao padrão de morbidade/ mortalidade. O presente estudo teve por objetivo descrever as mudanças do consumo alimentar em nipo-brasileiros de Bauru, no período de 1993 a 2000.

\section{Métodos}

\section{População de estudo}

A população amostral do presente estudo constituiu-se de 328 nipo-brasileiros residentes em Bauru, de primeira (issei) e segunda geração (nisei), de ambos os sexos, faixa etária de 40 a 79 anos em 1993, que participaram de dois inquéritos de saúde e nutrição realizados em 1993 e 2000. Nos dois inquéritos transversais, amostras sangüíneas de jejum foram obtidas para diagnóstico de alterações da homeostase glicêmica, conforme preconizado pela Organização Mundial da Saúde (OMS) 9. A dosagem de lipídeos séricos (triglicerídeos, colesterol total e HDL colesterol) foi realizada por procedimentos enzimáticos. Os valores de LDL e VLDL colesterol foram calculados em 1993; em 2000, os valores de LDL colesterol foram dosados e de VLDL colesterol calculados segundo fórmula proposta por Friedwald et al. 10. Em 1993 foi realizado estudo transversal de base populacional em japoneses de primeira e segunda geração, não-miscigenados, residentes em Bauru. Nesta fase inicial do estudo, 647 japoneses de primeira $(37,3 \%)$ e segunda geração $(62,7 \%)$, de ambos os sexos, foram submetidos ao primeiro inquérito de saúde e nutrição, conforme descrito em publicação anterior 11. Destes, 69 $(10,6 \%)$ foram a óbito, 57 (8,7\%) mudaram de endereço e 127 (19,7\%) recusaram-se a participar da segunda fase do projeto. No segundo inquérito transversal, 394 (60,9\% dos participantes em 1993) foram avaliados novamente em 2000. Para a presente análise, foram utilizados os dados de consumo alimentar individual de 328 indivíduos, uma vez que 67 participantes (17\% do total avaliado em 1993) não completaram as informações do inquérito nutricional.

\section{Avaliação antropométrica \\ e de consumo alimentar}

Medidas de peso (em quilogramas) e altura (em metros) foram obtidas em balança digital (capacidade $150 \mathrm{~kg}$, precisão $100 \mathrm{~g}$ ) e em estadiômetro fixo, respectivamente. O Índice de Massa Corporal (IMC) foi obtido dividindo-se o peso pela estatura ao quadrado $\left(\mathrm{kg} / \mathrm{m}^{2}\right)$ e a avaliação de sobrepeso e obesidade foi feita de acordo com os pontos de corte propostos pela OMS 12 .

Os dados de consumo alimentar em 1993 foram obtidos através de Questionário de Freqüência Alimentar (QFA) adaptado da versão original de Tsunehara et al. 13 com 177 itens alimentares. Em 2000, o QFA foi adaptado à análise em programa específico (Dietsys - National Cancer Institute, Bethesda, Maryland, Estados Unidos) para avaliação nutricional de dietas obtidas por questionários de freqüência alimentar, com dupla digitação dos dados dietéticos e análise de consistência para grande número de indivíduos, possibilitando a avaliação da dieta habitual segundo consumo de nutrientes e alimentos de forma rápida. Por esta razão, no inquérito nutricional realizado em 2000 a dieta habitual foi avaliada pelo QFA adaptado com 122 itens alimentares, com reprodutibilidade e validade previamente testados em nipobrasileiros de São Paulo 14. Nos dois inquéritos, a avaliação da dieta considerou o consumo habitual de alimentos e bebidas relativo ao período de um ano anterior ao momento da entrevista alimentar. Para padronização da avaliação do consumo alimentar nos dois inquéritos, os dados do QFA de 1993 foram transcritos para o modelo utilizado em 2000. A lista de alimentos e a freqüência de consumo utilizados no QFA de 1993 foram mantidos em 2000. Para classificação das porções alimentares do QFA de 1993 (obtidas como variável contínua em g ou $\mathrm{mL}$ ) utilizou-se a definição dos tamanhos das porções, como variável categórica (pequena, média, grande e extra-grande), conforme modelo adotado no QFA de 2000. Alguns alimentos com composição química semelhante e que constavam no QFA (1993) de forma isolada foram agrupados na versão do QFA (2000), somando-se suas freqüências de consumo em um mesmo item alimentar. Isto foi realizado para os seguintes alimentos: gobo (bardana) e nabo; macarronada, lasanha e outras massas; lingüiça e salsicha; lentilha, soja, feijão branco e grão de bico; batata, mandioca e inhame assados/cozidos; berinjela, chuchu e abobrinha refogados; queijo prato, mussarela, provolone e parmezão; uva, morango, pêssego e ameixa; pão francês, de forma, italiano e/ou sírio; pão 
integral, de trigo, centeio ou cevada. Outros alimentos com informação sobre consumo isolado no QFA (1993) (como gelatina, creme de leite, miso e leite de soja) foram considerados na análise quando apresentavam freqüência de consumo habitual de pelo menos uma vez por semana, tal como realizado no QFA (2000).

A descrição da dieta habitual da população de estudo analisou a participação relativa diária de grupos de alimentos e nutrientes selecionados. O consumo de nutrientes da dieta foi caracterizado como valores de proteínas, lipídeos, carboidratos e bebidas alcoólicas (em gramas e percentual do valor calórico total), calorias totais, percentual do valor calórico total proveniente dos doces, gordura saturada, ácido oléico, ácido linoléico, colesterol, cálcio, vitamina $\mathrm{C}$ e fibras. Os grupos de alimentos considerados (em gramas ou $\mathrm{mL}$ ) foram: verduras e legumes, frutas e sucos de frutas, leite e derivados e ovos.

A análise nutricional das dietas foi realizada com auxílio do programa Dietsys 4.01, com dados de composição química de alimentos compilados das tabelas oficiais do Brasil, Japão e Estados Unidos, conforme metodologia descrita em trabalho anterior 15,16.

\section{Análise estatística}

Para descrição das características gerais da amostra de estudo (indivíduos avaliados em 1993 e 2000) foram calculados médias, desviospadrão, freqüências relativas e absolutas. Foram utilizados os testes $t$ de Student para comparação entre amostras independentes ou teste de $\chi^{2}$ para comparação entre proporções.

Devido à distribuição não-Gaussiana dos dados de consumo de alguns nutrientes (de acordo com avaliação de simetria e curtose), medianas e intervalos interquartis (percentis 25 e 75) foram calculados para ingestão de nutrientes e alimentos nos dois períodos, segundo gênero. Não houve diferenças estatisticamente significantes no consumo alimentar segundo geração, tal como descrito em publicação anterior 16. Para comparação intra-indivíduos do consumo alimentar entre 1993 e 2000 foi utilizado o teste de Wilcoxon, com valor de $\mathrm{p}<0,05$. As análises estatísticas foram conduzidas no programa SPSS versão 10.0 (SPSS Inc., Chicago, Estados Unidos).

\section{Aspectos éticos}

O estudo foi aprovado pelo comitê de ética da Escola Paulista de Medicina, Universidade Federal de São Paulo, com consentimento escla- recido obtido de todos os participantes por escrito.

\section{Resultados}

A população amostral foi constituída por $48 \%$ de indivíduos do gênero masculino, 33\% de indivíduos de primeira geração, com idade média de 56,9 anos (desvio-padrão, DP = 9,8). Quando comparados os indivíduos que participaram dos dois inquéritos $(n=328)$ com os que não foram avaliados em $2000(\mathrm{n}=319)$, não houve diferença entre as proporções de homens (45\%) e indivíduos de primeira geração (29\%), com idade média de 57,7 anos ( $\mathrm{DP}=8,4)$.

A Tabela 1 apresenta as características gerais da população de estudo em 1993 e 2000, segundo gênero. A proporção de indivíduos de primeira e segunda geração (Issei/Nisei, respectivamente) foi semelhante entre os sexos. Em 1993, os valores percentuais de tabagismo, sobrepeso/obesidade, diabetes mellitus, dislipidemias e valores médios de idade e IMC foram semelhantes entre homens e mulheres. No entanto, maiores freqüências de consumo habitual de álcool e maior nível de escolaridade foram observados entre os homens. No período de 1993 a 2000 foram detectados setenta novos casos de diabetes na amostra estudada (quarenta casos novos entre os homens e trinta entre as mulheres).

A Tabela 2 apresenta os valores medianos (intervalos interquartis) do consumo de nutrientes e alimentos em 1993 e 2000, segundo gênero. O consumo calórico no período situou-se na faixa da recomendação específica para japoneses em ambos os gêneros 17. No entanto, a contribuição calórica percentual das gorduras foi acima do recomendado pela OMS 12 (30\% das calorias totais) e para a população japonesa 17 (20-25\% calorias totais). O consumo de proteínas (em gramas) foi acima dos valores recomendados pela OMS 18 (cerca de 60g/dia) e dentro do intervalo recomendado para a população japonesa (70-85g/dia). Para a vitamina C, a ingestão foi bem acima do recomendado para japoneses, pela OMS 18 e do recomendado para a população americana 19 nos dois períodos (valores recomendados, respectivamente: $50 \mathrm{mg}$, 50-60mg e $75 \mathrm{mg}$ para mulheres e $90 \mathrm{mg}$ para homens). Por outro lado, o consumo de cálcio foi abaixo do recomendado pela OMS 18 e pelo Institute of Medicine dos Estados Unidos 19 nos dois períodos (valores recomendados de 500-800mg e 1.000-1.200mg, respectivamente). $O$ consumo de fibras foi também abaixo do recomendado para a população japone- 
Características gerais da amostra de estudo, segundo gênero em 1993 e 2000. Bauru, São Paulo, Brasil.

\begin{tabular}{|c|c|c|c|c|}
\hline & \multicolumn{2}{|c|}{1993} & \multicolumn{2}{|c|}{2000} \\
\hline & $\begin{array}{l}\text { Mulheres } \\
(n=169)\end{array}$ & $\begin{array}{l}\text { Homens } \\
(n=159)\end{array}$ & $\begin{array}{l}\text { Mulheres } \\
(n=169)\end{array}$ & $\begin{array}{l}\text { Homens } \\
(\mathrm{n}=159)\end{array}$ \\
\hline Proporção Issei/Nisei & 0,48 & 0,50 & 0,48 & 0,50 \\
\hline Idade média (DP) em anos & $57,1(9,8)$ & $56,6(9,8)$ & $63,7(9,9)$ & $63,2(9,9)$ \\
\hline Índice de Massa Corporal médio (DP) em kg/m² & $24,6(3,7)$ & $24,8(3,7)$ & $24,6(3,6)$ & $25,0(3,7)$ \\
\hline Sobrepeso/Obesidade (\%) & 41,5 & 44,2 & 44,4 & 44,7 \\
\hline Diabetes mellitus (\%) & 21,8 & 19,2 & 39,6 & 44,7 \\
\hline Dislipidemias (\%) & 87,5 & 90,5 & 91,7 & 89,9 \\
\hline Tabagismo (\%) & 20,7 & 16,3 & 13,0 & 8,2 \\
\hline Consumo habitual de álcool (\%)* & 12,4 & 59,7 & 16,6 & 56,6 \\
\hline \multicolumn{5}{|l|}{ Escolaridade (\%)* } \\
\hline Não freqüentou escola & 17,3 & 15,1 & 17,3 & 15,1 \\
\hline Até 1o grau & 58,9 & 37,7 & 58,9 & 37,7 \\
\hline Até 2o grau & 11,9 & 17,0 & 11,9 & 17,0 \\
\hline Universitário & 11,9 & 30,2 & 11,9 & 30,2 \\
\hline
\end{tabular}

* Diferenças segundo gênero estatisticamente significantes, $p=0,000$.

Valores medianos (intervalo-interquartil) do consumo diário de energia, macronutrientes e alimentos selecionados em nipo-brasileiros em 1993 e 2000. Bauru, São Paulo, Brasil.

\begin{tabular}{|c|c|c|c|c|}
\hline \multirow[t]{2}{*}{ Nutrientes/Alimentos } & \multicolumn{2}{|c|}{ Mulheres $(n=169)$} & \multicolumn{2}{|c|}{ Homens ( $n=159$ ) } \\
\hline & 1993 & 2000 & 1993 & 2000 \\
\hline Calorias totais* em Kcal & $1.581,3(1.262,1-1.963,5)$ & $1.754,7(1.354,6-2.065,9)^{\star \star}$ & $1.945,2(1.020,0-2.487,0)$ & $2.027,3(1.695,6-2.485,5)$ \\
\hline Proteínas (g) & $63,3(48,7-79,3)$ & $61,9(49,9-75,5)$ & $75,1(50,0-95,9)$ & $72,2(53,8-90,4)^{\star \star}$ \\
\hline$\%$ calorias totais* & $15,7(13,5-17,9)$ & $14,4(12,5-16,1)^{\star \star}$ & $15,7(13,3-18,6)$ & $13,6(12,0-15,7)^{\star \star}$ \\
\hline Lipídeos totais (g) & $48,2(38,0-62,0)$ & $61,4(48,1-74,6)^{\star \star}$ & $56,9(42,2-69,8)$ & $68,8(52,6-89,2)^{\star \star}$ \\
\hline$\%$ calorias totais & $27,7(24,0-32,0)$ & $31,5(27,4-36,6)^{\star \star}$ & $26,0(21,6-30,4)$ & $30,8(27,0-35,0)^{\star \star}$ \\
\hline Carboidratos (g) & $222,7(179,2-287,6)$ & $228,6(176,4-290,3)$ & $271,8(217,1-328,0)$ & $276,3(225,2-324,0)$ \\
\hline$\%$ calorias totais & $57,8(52,7-62,5)$ & $54,9(49,2-60,8)^{\star \star}$ & $54,8(47,6-63,0)$ & $53,8(48,0-59,8)$ \\
\hline Gordura saturada (g) & $15,6(10,8-19,4)$ & $14,4(10,2-18,4)$ & $16,8(12,3-22,3)$ & $16,9(12,6-22,5)$ \\
\hline Ácido graxo oléico (g) & $15,2(12,3-21,7)$ & $22,5(17,3-27,4)^{\star \star}$ & $18,3(13,4-24,0)$ & $25,5(19,3-33,0)^{\star \star}$ \\
\hline Ácido graxo linoléico (g) & $6,8(5,0-10,1)$ & $9,7(7,3-12,5)^{\star \star}$ & $7,8(4,0-10,6)$ & $10,5(8,0-14,2)^{\star \star}$ \\
\hline Colesterol (mg) & $200,3(134,8-273,7)$ & $151,7(106,8-199,4)^{\star \star}$ & $238,2(157,7-352,7)$ & $192,4(124,1-264,4)^{\star \star}$ \\
\hline Fibras (g) & $15,8(10,7-21,3)$ & $15,6(11,9-20,6)$ & $16,9(12,4-22,3)$ & $17,8(13,9-22,5)$ \\
\hline Cálcio (mg) & $571,9(448,9-719,4)$ & $669,7(519,2-836,8)^{*}$ & $604,9(452,7-803,1)$ & $680,9(492,7-886,4)^{\star}$ \\
\hline Vitamina C (mg) & $202,5(132,0-278,0)$ & $204,2(139,1-304,4)^{*}$ & $175,7(115,7-270,8)$ & $204,3(135,9-287,1)$ \\
\hline Verduras e legumes (g) & $220,6(150,6-320,4)$ & $188,6(134,2-230,3)^{*}$ & $206,2(145,5-294,2)$ & $203,3(143,1-266,3)$ \\
\hline Frutas e sucos $(\mathrm{g} / \mathrm{ml})$ & $273,7(175,0-460,7)$ & $358,1(232,7-529,8)^{*}$ & $254,4(160,7-432,4)$ & $389,3(211,5-429,9)^{*}$ \\
\hline Laticínios totais ( $\mathrm{g} / \mathrm{ml})$ & $156,7(55,7-192,8)$ & $177,1(135,0-258,5)^{\star}$ & $161,6(51,8-2 \mathrm{~s} 12,9)$ & $174,8(84,3-230,0)^{*}$ \\
\hline Ovos $(\mathrm{g})$ & $8,6(4,0-17,1)$ & $8,6(3,0-17,1)$ & $19,2(5,0-64,6)$ & $17,1(5,9 ; 47,5)^{\star}$ \\
\hline
\end{tabular}

* Incluindo as calorias provenientes de álcool;

** Teste de Wilcoxon: $p<0,05$. 
sa (20g/dia) e dentro do intervalo recomendado pela OMS 18 (15-20g/dia) em 1993 e 2000. De acordo com as recomendações dietéticas para a população japonesa, observou-se, em ambos os gêneros, consumo mediano de frutas adequado nos dois períodos. No entanto, o consumo mediano de verduras e legumes, ovos e grupo dos leites e derivados encontrou-se cerca de $32 \%, 83 \%$ e $34 \%$, respectivamente, abaixo do recomendado nos dois períodos (recomendação para a população japonesa: 300g, 40-50g e 200-300g, respectivamente).

Em geral, as alterações do consumo de nutrientes foram semelhantes entre os gêneros. No entanto, entre as mulheres houve aumento do valor calórico total das dietas com redução do percentual calórico para doces. Entre os homens, houve redução do consumo em gramas de proteínas. Em ambos os gêneros, houve redução na contribuição percentual calórica das proteínas e aumento deste percentual proveniente das gorduras. Entre os componentes dos lipídeos ingeridos, não houve alteração no consumo de gordura saturada, mas houve aumento no consumo de ácidos graxos mono e polinsaturados (oléico e linoléico) e redução do colesterol. A ingestão de cálcio aumentou, sem alteração no consumo de vitamina $\mathrm{C}$ e fibras. Em relação ao consumo de grupos de alimentos, não houve alteração na ingestão de verduras e legumes entre os homens, mas houve redução entre as mulheres. Em ambos os gêneros, o consumo de frutas e sucos de frutas e de laticínios aumentou. O consumo de ovos não se alterou entre as mulheres, mas se reduziu entre os homens.

\section{Discussão}

Apesar do aumento no consumo total de gordura da dieta, as mudanças no consumo alimentar observadas entre os nipo-brasileiros de Bauru sugerem tendência à adesão a dietas mais saudáveis, como redução nos teores de colesterol e aumento no consumo de ácidos graxos polinsaturados, frutas e cálcio. No entanto, observou-se consumo abaixo das recomendações internacionais para cálcio e vegetais em geral.

No presente estudo, os níveis de ingestão de gordura total foram superiores aos observados na dieta habitual de japoneses residentes no Japão e inferiores ao consumo de nipo-americanos do Havaí e Califórnia 1. O aumento do consumo de gorduras totais da dieta pode ser atribuído ao maior consumo de gorduras insaturadas, uma vez que a ingestão de gordura sa- turada não se alterou no período do estudo. Houve também redução no consumo de colesterol, cujos valores medianos foram inferiores ao consumo médio estimado para nipo-brasileiros de São Paulo (457mg) 5, japoneses residentes em diferentes regiões do Japão (entre 339 a $487 \mathrm{mg}$ ) 20 e para nipo-americanos (cerca de 540mg) 1. Aumento no consumo de cálcio, laticínios e frutas/sucos de frutas, em ambos os gêneros, foram também observados entre os nipo-brasileiros de Bauru. Apesar do consumo abaixo do recomendado para verduras e legumes, ovos e laticínios, os valores medianos observados foram superiores aos encontrados em japoneses residentes no Japão 20 e semelhantes ao consumo de nipo-brasileiros da cidade de São Paulo 5. Para o consumo de frutas e sucos de frutas, no entanto, os valores medianos observados entre os nipo-brasileiros de Bauru estavam acima das recomendações nutricionais e foram semelhantes aos encontrados entre nipo-brasileiros de São Paulo 5.

Nossos resultados apresentam algumas semelhanças e diferenças com os encontrados em Pesquisas de Orçamentos Familiares (POF) na população geral brasileira realizados no período de 1988-1996 e de 2002-2003 em áreas metropolitanas no Brasil 21,22. Na região do centro-sul do país observou-se aumento no consumo de leite e derivados e redução no consumo de ovos (observado entre homens em nosso estudo). Consumo insuficiente de verduras e legumes foi também apontado, semelhante ao presente estudo. O consumo percentual de calorias provenientes das gorduras ultrapassou os valores recomendados pela OMS 12 em nossa população de estudo e foi cerca de $2 \%$ maior que o consumo estimado para a população brasileira em geral da região urbana ${ }^{22}$. Já em relação ao consumo total de frutas, enquanto a população brasileira em geral parece consumir menos que o recomendado para este grupo de alimentos 22 , os nipo-brasileiros referem consumo habitual freqüente.

Essas características do padrão alimentar foram recentemente analisadas em relação ao perfil de risco para doenças crônicas não transmissíveis em nipo-brasileiros de Bauru: Freire et al. 6 encontraram aumento do risco para síndrome metabólica entre indivíduos com maior consumo de gordura total, porém com efeito protetor para o maior quintil de consumo de ácido graxo linoléico, confirmando as recomendações internacionais com relação não somente aos limites máximos de ingestão de gordura total mas também à qualidade da gordura ingerida; Sartorelli et al. 7 apontaram maior risco para distúrbios da homeostase glicêmica (gli- 
cemia de jejum alterada e/ou intolerância à glicose diminuída) entre os nipo-brasileiros que referiram maior consumo de fibras provenientes essencialmente de frutas e sucos de frutas, pão branco e arroz polido (mediana do maior tercil de consumo diário foi cerca de $1 \mathrm{~kg}$ para este grupo de alimentos).

Em estudos prospectivos, o impacto do efeito de seguimento da população de estudo deve ser considerado na adoção ou no relato de hábitos alimentares e de saúde em geral. Desta forma, o diagnóstico de diabetes mellitus e doenças associadas, realizado em 1993 em nosso estudo pode ter influenciado as informações de consumo alimentar habitual. Ainda assim, as alterações do padrão de consumo alimentar descritas aqui foram consistentes com os resultados das análises transversais do inquérito realizado em 2000, que incluíram somente casos novos de distúrbios da homeostase glicêmica 7 e síndrome metabólica 6 .

As adaptações realizadas no QFA utilizado no inquérito conduzido em 2000 podem representar uma das limitações do presente estudo. O QFA utilizado em 1993 não foi previamente testado quanto a sua validade e reprodutibilidade na comunidade nipo-brasileira. No entanto, em 2000, o QFA foi previamente testado com grau de confiabilidade e acurácia adequado para este tipo de questionário 14. Estudos prévios demonstraram que a lista de alimentos e freqüência de consumo de um QFA são os principais determinantes da estimativa de consumo alimentar total em inquéritos epidemiológicos 8 , sugerindo desempenho aceitável do QFA no presente estudo, uma vez que a lista de alimentos e freqüência de consumo foram semelhantes nos QFA utilizados nos dois inquéritos.

No presente estudo, cerca de $50 \%$ dos indivíduos inicialmente avaliados na amostra populacional de 1993 foram re-avaliados em 2000, representando um vício potencial no estudo com seleção de indivíduos mais saudáveis ou motivados à participação, impossibilitando a extrapolação desses resultados para toda a comunidade nipo-brasileira de Bauru. No entanto, quando comparados os indivíduos que participaram dos dois inquéritos $(n=328)$ com os que não foram avaliados em 2000 ( $n=319)$, não houve diferenças estatisticamente significantes segundo gênero, geração e idade.

Com base em evidências do papel da ingestão de frutas e vegetais na prevenção de doenças cardiovasculares pela combinação benéfica de micronutrientes 23,24, a American Heart Association (AHA) recomenda o consumo de 5 ou mais porções de frutas e vegetais por dia 25 , o que corresponde, aproximadamente, a 400g/ dia destes alimentos. No Japão, onde o consumo habitual de frutas frescas ainda é muito baixo, um estudo de casos e controles encontrou que o consumo de mais de duas porções de frutas por semana pode reduzir o risco de infarto agudo do miocárdio em homens e mulheres 26 .

De acordo com os resultados do presente estudo e de outras publicações recentes sobre padrão alimentar de nipo-brasileiros de Bauru e conforme recomendações preconizadas pela OMS para prevenção de doenças crônicas não transmissíveis, a adoção de estilo de vida saudável (não fumar, manter-se fisicamente ativo e controle de peso) deve ser urgentemente implementada nessa população, destacando-se as seguintes recomendações dietéticas: (a) distribuição das calorias provenientes das gorduras da dieta: menos de $10 \%$ de ácidos graxos saturados (incluindo as formas trans) e mínimo de $20 \%$ de ácidos graxos mono- e poli-insaturados; (b) incentivo ao consumo de alimentos naturalmente ricos em fibras e micronutrientes como cereais integrais, verduras e legumes frescos, preferencialmente. 


\section{Resumo}

Foram estudados 328 nipo-brasileiros, ambos os sexos e faixa etária de 40 a 79 anos em 1993, participantes de dois inquéritos de saúde e nutrição realizados em 1993 e 2000. A avaliação da dieta foi realizada por questionários quantitativos de freqüência. A análise das alterações da dieta entre 1993 e 2000, segundo sexo, utilizou o teste de Wilcoxon para amostras dependentes ( $p<0,05)$. Em ambos os sexos, quando comparadas diferenças de consumo alimentar nos dois inquéritos, houve aumento da contribuição do consumo de gorduras para as calorias totais em 16\% (valores médios em 2000: 31,5\% para mulheres e 30,8\% para homens). Houve aumento de $40 \%$ no consumo de ácidos graxos oléico e linoléico, com redução de $20 \%$ no colesterol. Entre as mulheres, observou-se aumento nas calorias totais, redução no consumo de vegetais e percentual calórico dos doces. Apesar do aumento do consumo de gordura total da dieta, houve redução no teor de colesterol e aumento no consumo de ácidos graxos linoléico e oléico, frutas e cálcio. Ainda assim, os níveis de ingestão de cálcio e vegetais foram abaixo das recomendações internacionais.

Consumo de Alimentos; Nutrição; Inquéritos Nutricionais

\section{Colaboradores}

T. G. Castro organizou os bancos de dados dos dois inquéritos, revisão da literatura, análise dos resultados e redação do manuscrito. C. N. Bertolino participou do processamento e análise dos dados. S. G. A. Gimeno desenvolveu o trabalho de campo juntamente com o Grupo de Estudos de Diabetes de Nipo-Brasileiros de Bauru e colaborou nas análises estatísticas dos dados. M. A. Cardoso desenvolveu o trabalho de campo, planejou o estudo e elaborou a versão final do manuscrito.

\section{Outros membros do Grupo de Estudos de Diabetes em Nipo-Brasileiros}

N. Barros Jr., R. Chaim, V. D'Almeida, S. R. G. Ferreira, L. J. Franco, R. D. Freire, H. Harima, A. Hirai, A. T. Hirai, M. Iunes (in memorian), M. Kikuchi, L. Matsumura, R. S. Moisés, K. Osiro, N. Tomita, K. Wakisaka.

\section{Agradecimentos}

Este trabalho teve o apoio da Fundação de Amparo à Pesquisa do Estado de São Paulo que concedeu bolsa de mestrado à T. G. Castro (processo n. 01/11391-3R).

\section{Referências}

1. Kagan A, Harris BR, Winkelstein Jr. W, Johnson KG, Kato H, Syme SL, et al. Epidemiologic studies of coronary heart disease and stroke in Japanese men living in Japan, Hawaii and California: demographic, physical, dietary and biochemical characteristics. J Chronic Dis 1974; 27:345-64.

2. Gimeno SGA, Ferreira SRG, Franco LJ, Hirai AT, Matsumura L, Moisés RS. Prevalence and 7-year incidence of type 2 diabetes mellitus in a JapaneseBrazilian population: an alarming public health problem. Diabetologia 2002; 45:1635-8.

3. Tsumura K, Hayashi T, Suematsu C, Endo G, Fujii S, Okada K. Daily alcohol consumption and the risk of type 2 diabetes in Japanese men: the Osaka Health Survey. Diabetes Care 1999; 221:14327.

4. Malerbi DA, Franco LJ. Multicenter study of the prevalence of diabetes mellitus and impaired glucose tolerance in the urban Brazilian population aged 30-69 yr. The Brazilian Cooperative Group on the Study of Diabetes Prevalence. Diabetes Care 1992; 15:1509-16.

5. Cardoso MA, Hamada GS, Souza JMP, Tsugane S, Tokudome S. Dietary patterns in Japanese migrants to southearstern Brazil and their descendants. J Epidemiol 1997; 7:198-204.

6. Freire RD, Cardoso MA, Gimeno SGA, Ferreira SRG; Japanese-Brazilian Diabetes Study Group. Dietary fat is associated with metabolic syndrome in Japanese-Brazilians. Diabetes Care 2005; 28: 1779-85.

7. Sartorelli DS, Freire RD, Ferreira SRG, Cardoso MA; Japanese-Brazilian Diabetes Study Group. Dietary fiber and glucose tolerance in JapaneseBrazilians. Diabetes Care 2005; 28:2240-2.

8. Willett WC, Lenart E. Reproducibility and validity of food frequency questionnaires. In: Willett WC, editor. Nutritional epidemiology. 2nd Ed. New York: Oxford University Press; 1998. p. 101-47.

9. Alberti KGMM, Zimmet PZ; WHO Consultation. Definition, Diagnosis and Classification of Diabetes Mellitus and its Complications. Part 1: Diagnosis and Classification of Diabetes Mellitus. Provisional Report of a WHO Consulation. Diabet Med 1999; 15:539-53.

10. Friedwald W, Levy RJ, Friedrickson DS. Estimation of the concentration of low-density lipoprotein cholesterol in plasma, without use of the preparative ultracentrifuge. Clin Chem 1972; 18: 499-502.

11. Gimeno SGA, Ferreira SRG, Cardoso MA, Franco LJ, Iunes M; Japanese-Brazilian Diabetes Study Group. Weight gain in adulthood and risk of developing glucose tolerance disturbance: a study of a Japanese-Brazilian population. J Epidemiol 2000; 10:103-10.

12. World Health Organization. Diet, nutrition and the prevention of chronic diseases: report WHO/FAO. Geneva: World Health Organization; 2003. (Technical Report Series, 916).

13. Tsunehara C, Leonetti DL, Fujimoto WY. Diet of second-generation Japanese-American men with and without non-insulin-dependent diabetes. Am J Clin Nutr 1990; 52:731-8. 
14. Cardoso MA, Kida AA, Tomita LY, Stocco PR. Reproducibility and validity of a food frequency questionnaire among women of Japanese ancestry living in Brazil. Nutr Res 2001; 21:725-33.

15. Cardoso MA, Stocco PR. Desenvolvimento de um questionário quantitativo de freqüência alimentar em imigrantes japoneses e seus descendentes residentes em São Paulo, Brasil. Cad Saúde Pública $2000 ; 16: 107-14$

16. Freire RD, Cardoso MA, Shinzato AR, Ferreira SRG; The Japanese-Brazilian Diabetes Study Group. Nutritional status of Japanese-Brazilians: comparison across sex and generation. Br J Nutr 2003; 89:705-12.

17. Ministry of Health and Welfare. Recommended dietary allowances for the Japanese. 5th Revision. Tokyo: Daiicchi Shuppan; 1996.

18. Organización Mundial de la Salud. Preparación y uso de diretrices nutricionales basadas em los alimentos: informe FAO-OMS. Geneva: Organización Mundial de la Salud; 1998. (Serie de Informes Técnicos, 880).

19. Food and Nutrition Board, Institute of Medicine/ National Academy of Sciences Dietary Reference Intake. Recommended intakes for individuals. Washington DC: National Academy Press; 2000.

20. Ueshima H, Iida M, Shimamoto T, Konishi M, Tanigaki M, Doi M, et al. Dietary intake and serum total cholesterol level: their relationship to different lifestyles in several Japanese populations. Circulation 1982; 66:519-26.
21. Monteiro CA, Mondini L, Costa RBL. Mudanças na composição e adequação nutricional da dieta familiar nas áreas metropolitanas do Brasil (19881996). Rev Saúde Pública 2000; 34:251-8.

22. Instituto Brasileiro de Geografia e Estatística. Pesquisa de Orçamentos Familiares 2002-2003. Rio de Janeiro: Instituto Brasileiro de Geografia e Estatística; 2003.

23. Liu S, Manson JE, Lee I, Cole SR, Hennekens CH, Willett WC, et al. Fruit and vegetable intake and risk of cardiovascular disease: the Women's Health Study. Am J Clin Nutr 2000; 72:922-8.

24. Liu S, Lee I, Ajani U, Cole SR, Buring JE, Manson JE. Intake of vegetables rich in carotenoids and risk of coronary heart disease in men: The Physician's Health Study. Int J Epidemiol 2001; 30:130-5.

25. American Heart Association. AHA dietary guidelines: revision 2000. A statement for healthcare professionals from the nutrition committee of the American Heart Association. Circulation 2000; 102:2284-99.

26. Sasazuki S; Fukuoka Heart Study Group. Casecontrol study of nonfatal myocardial infarction in relation to selected foods in Japanese men and women. Jpn Circ J 2001; 65:200-6.

Recebido em 30/Set/2005

Aprovado em 10/Mar/2006 